Elsevier required licence: (C) <year>. This manuscript version is made available under the CC-BYNC-ND 4.0 license http://creativecommons.org/licenses/by-nc-nd/4.0/ 


\title{
Lagrangian Relaxation versus Genetic Algorithm based Matheuristic for a Large Partitioning Problem
}

\author{
Oliver G. Czibula, Hanyu Gu, Yakov Zinder \\ School of Mathematical and Physical Sciences, University of Technology Sydney 15 \\ Broadway Ultimo NSW 2007, Australia
}

\begin{abstract}
This paper is concerned with a partitioning problem. One of the applications, and the motivation for this research, is the problem of class formation for training and retraining sessions at large electricity distributors. Two different approaches are developed. One is based on the Quadratic Multiple Knapsack formulation and Lagrangian relaxation. The other is a matheuristic developed as an amalgamation of Genetic Algorithms and Integer Programming. The approaches are tested by means of computational experiments. Both heuristics outperformed the direct application of quadratic programming, with the Lagrangian relaxation based approach performing the best on average, and the Genetic Algorithm based approach performing the best on the larger test cases. Keywords: Partitioning problem, Integer programming, Lagrangian relaxation, Genetic algorithm, Heuristic
\end{abstract}

\section{Introduction}

This paper is concerned with the combinatorial optimisation problem where the union of disjoint sets $F_{1}, \ldots, F_{K}$ (referred to as families) must be partitioned into sets $S_{1}, \ldots, S_{N}$ in such a way that each $S_{i}$ is either empty, or has cardinality

between $a_{i}$ and $b_{i}$, i.e. $a_{i} \leq\left|S_{i}\right| \leq b_{i}$, and each nonempty set $S_{i}$ must contain elements from at least $p_{i}$ and at most $q_{i}$ families. All $a_{i}, b_{i}, p_{i}$, and $q_{i}$ are given.

There is a cost $c_{i, j}$ associated with the inclusion of each element $j$ of $F_{1} \cup$ $\ldots \cup F_{n}$ into each set $S_{i}$. For any two distinct families $F_{k}$ and $F_{l}$, and any set 
$S_{i}$, the cost $b_{k, l}$ is incurred for the simultaneous presence of elements from both families $F_{k}$ and $F_{l}$ in $S_{i}$. This cost does not depend on the number of elements from $F_{k}$ and $F_{l}$ in $S_{i}$. The objective is to minimise the total cost associated with the partition $S_{1}, \ldots, S_{N}$.

The above mentioned combinatorial optimisation problem was motivated in particular by the problem of class formation for training and retraining at large electricity distributors. Such organisations typically have thousands of workers of different types, and some also provide training to their contractors and to third parties. Due to the multitude of hazards that exist when working with high voltages, at heights, or in confined spaces, local laws often require all such workers to undergo regular safety, technical, and professional training.

Many of these workers, referred to as students for the purpose of this study, have very different learning outcomes from these courses, different learning styles, different levels of education or English proficiency, and different levels of technical proficiency for certain tasks. Although assigning only one type of student into any class enables the training delivery to be better tailored to the needs of the specific group, in some cases it is beneficial to combine several compatible types of students in the same class to facilitate the exchange of knowledge and experience between student types. Furthermore, due to the cost of delivering training and the scarcity of training resources, it is often not possible to run segregated classes, and some undesirable blending of student

so types may be necessary. Each class can either be empty, in which case it will be cancelled with no penalty, otherwise it must contain between a minimum and a maximum number of students, and between a minimum and maximum number of student types.

Each potential class is associated with a specific date at which it will be 35 conducted. On the other hand, each student, who is of exactly one type, should complete their training by a certain date, resulting in a penalty (cost) incurred for assigning a student to a particular class. Another type of penalty reflects the incompatibility of the student types assigned to the same class. The objective is to minimise the total penalty. When a single course is considered, the above 
leads to the combinatorial optimisation problem studied in this paper. We denote the problem studied in this paper to be "Partition into Inhomogeneous Classes" (PIC).

The considered combinatorial optimisation problem is distinct from, but shares certain similarity with, the Graph Partitioning Problem (GPP) [1]. This

45 problem arises in various practical situations, for example: partitioning subroutines that are compiled into computer code into clusters, such that the communication between clusters is minimised [2]; assigning data or processes evenly to processors, such that inter-process communication is minimised in parallel computing environments [3]; in aircraft control, where the flow of aircraft within blocks is maximised and flow of aircraft between blocks is minimised [4; partitioning the electronic subcircuits in very large-scale integration (VLSI) systems, such that the electrical connections between partitions is minimised [5]; partitioning a power network into so-called islands to prevent the propagation of cascading failures [6], 7]; for route planning along road networks [8, 9]; and in many other domains. Although the combinatorial optimisation problem considered in our paper and the GPP are different, each provides an insight in the design of solution techniques for the other.

Several particular cases of the GPP, including Integer Programming (IP) models for each case, are discussed in [10]. The authors take advantage of the graph structure when clustering. They also discuss several valid inequalities and facet-defining inequalities for the GPP. A column generation approach is presented in [2], which the authors tested on graphs with between 30 and 61 nodes, and between 47 and 187 edges. For the 12 test cases the authors considered, their proposed approach provided integer solutions for all but two, and for those, solutions obtained by a branch-and-bound scheme were very close to the fractional solutions provided by column generation. Our paper also presents IP models for the considered partitioning problem, but is concerned with a Lagrangian relaxation and a matheuristic, and is focussed on solving large instances with hundreds of classes and thousands of students.

The problem considered in our paper is also related to the Quadratic Multi- 
ple Knapsack Problem (QMKP), which is a combination of the multiple knapsack and quadratic knapsack problems. The QMKP received little attention in the literature until recently, and most solution approaches are based on metaheuristics [11, 12], 13], 14]. In particular, greedy and genetic algorithms (GA) 75 for the QMKP are discussed in [14]. The author presents two greedy heuristics that build solutions by choosing objects according to their value densities, and two GA heuristics. One GA is a standard implementation, while the other is extended with greedy techniques that probabilistically favour objects of high value density. The algorithms are tested on 20 problem instances, and the extended GA is reported to perform best on all but one test case. In our paper we present a matheuristic that is an amalgamation of Genetic Algorithms and IP.

A Langrangian relaxation based approach to solving the QMKP exactly is presented in [15], whereby a tighter bound is computed using the subgradient method. The proposed approach is able to optimally solve instances with up to 400 binary variables. In our paper we also consider a Langrangian relaxation based approach, but focus on problems with between 17697 and 267393 binary variables (and between 42656 and 367232 for the linearised models). The size of our problem, together with the complexity results presented in our paper, leads 90 to the aim of obtaining a good approximation solution, which is compared with the alternative approach that is an amalgamation between Genetic Algorithms and IP.

The considered problem was previously studied in [16]. In contrast to [16] our current paper is focused on significantly larger test cases, which has greater significance for practical applications. The change of focus necessitated the revision of the proposed approaches, resulting in superior performance of the designed algorithms on the larger test cases.

The remainder of this paper is organised as follows. Section 2 presents a linearly constrained quadratic programming formulation, as well as its lineari100 sation, for the studied problem. Section 3 analyses the complexity of the considered partitioning problem. Section 4 discusses a Lagrangian relaxation of the 
quadratic program. Section 5 presents the first approach, which is a heuristic based on the Lagrangian relaxation. Section 6 presents the second approach, which is an amalgamation of Genetic Algorithms and Integer Programming. Section 7 discusses the results of the computational experimentation. Our concluding remarks are given in Section 8

\section{Quadratic Programming Formulation}

Let $\mathcal{N}=\{1, \cdots, N\}, \mathcal{M}=\{1, \cdots, M\}$, and $\mathcal{K}=\{1, \cdots, K\}$ be the set of available classes, the set of students to be assigned, and the set of student types respectively. Denote the penalty of assigning student $j \in \mathcal{M}$ to class $i \in \mathcal{N}$ by $c_{i, j}$, and the penalty of pairing student types $k \in \mathcal{K}$ and $l \in \mathcal{K}$ together in the same class by $b_{k, l}$. Each student has exactly one type, and the set of students who are of type $k$ is represented by $T_{k}, k \in \mathcal{K}$. Each student must be assigned to exactly one class, but not all classes must be run. Each class $i \in \mathcal{N}$ that is run must contain at least $a_{i}$ and at most $b_{i}$ students, and at least $p_{i}$ and at most $q_{i}$ student types. Students or student types cannot be assigned to classes that are not run. The binary variable $X_{i, j}$ is defined to be 1 if student $j$ is assigned to class $i$, or 0 otherwise; the binary variable $Y_{i, k}$ is defined to be 1 if student type $k$ is assigned to class $i$, or 0 otherwise; The binary variable $Z_{i}$ is defined to be 1 if class $i$ is run, or 0 otherwise. The following Quadratic Program describes the problem:

$$
\text { (QP) Minimise: } \alpha \sum_{i=1}^{N} \sum_{k=1}^{K} \sum_{l=1}^{K} b_{k, l} Y_{i, k} Y_{i, l}+\beta \sum_{i=1}^{N} \sum_{j=1}^{M} c_{i, j} X_{i, j}
$$




$$
\begin{array}{rl}
\text { Subject To: } \quad \sum_{i=1}^{N} X_{i, j}=1 \quad j=1, \ldots, M \\
a_{i} Z_{i} \leq \sum_{j=1}^{M} X_{i, j} \leq b_{i} Z_{i} & i=1, \ldots, N \\
p_{i} Z_{i} \leq \sum_{k=1}^{K} Y_{i, k} \leq q_{i} Z_{i} & i=1, \ldots, N \\
X_{i, j} \leq Y_{i, k} & i=1, \ldots, N ; k=1, \ldots, K ; j \in T_{k} \\
Y_{i, k} \leq \sum_{j \in T_{k}} X_{i, j} & i=1, \ldots, N ; k=1, \ldots, K \\
X_{i, j} \in\{0,1\} & i=1, \ldots, N ; j=1, \ldots, M \\
Y_{i, k} \in\{0,1\} & i=1, \ldots, N ; k=1, \ldots, K \\
Z_{i} \in\{0,1\} & i=1, \ldots, N
\end{array}
$$

The quadratic term in (11) represents the penalty of pairing student types together, and the linear term represents the penalty of assigning students to classes, weighted by coefficients $\alpha$ and $\beta$, respectively.

The constraints (2) express the requirement that each student must be assigned to exactly one class. The constraints (3) and (4) express the requirement that each running class must have between $a_{i}$ and $b_{i}$ students, and between $p_{i}$ and $q_{i}$ student types, respectively, if the class is run, or zero otherwise. The constraints (5) express the requirement that a student may only be assigned to a class if that student's type has also been assigned to that class. The constraints (6) ensure that a class can only be assigned a type if at least one student of that type is in the class.

It is possible to linearise the quadratic term in (1) by introducing $\hat{Y}_{i, k, l}=$ $Y_{i, k} Y_{i, l}$ together with constraints:

$$
\begin{array}{r}
\hat{Y}_{i, k, l} \leq Y_{i, k} \quad i=1, \ldots, N ; k=1, \ldots, K ; l=1, \ldots, K \\
\hat{Y}_{i, k, l} \leq Y_{i, l} \quad i=1, \ldots, N ; k=1, \ldots, K ; l=1, \ldots, K \\
\hat{Y}_{i, k, l} \geq Y_{i, k}+Y_{i, l}-1 \quad i=1, \ldots, N ; k=1, \ldots, K ; l=1, \ldots, K
\end{array}
$$


to give the linearised model:

$$
\begin{array}{r}
\text { (LQP) Minimise: } \alpha \sum_{i=1}^{N} \sum_{k=1}^{K} \sum_{l=1}^{K} b_{k, l} \hat{Y}_{i, k, l}+\beta \sum_{i=1}^{N} \sum_{j=1}^{M} c_{i, j} X_{i, j} \\
\text { Subject To: }-21, \ldots, K ; l=1, \ldots, K
\end{array}
$$

The time required to find an optimal solution to the (QP) and (LQP) models grows rapidly, where even some small test cases with just a few dozen students can take hours to solve. As the problem instances we hope to solve are significantly larger than this, we propose to use a heuristic approach.

\section{NP-completeness}

This section presents a proof of the NP-completeness in the strong sense of the following problem:

\section{C_PARTITION}

Input: a set $S$; a function $c$ that associates with each pair $\{j, g\} \subset S$ of distinct elements a nonnegative integer $c(j, g)$; a nonnegative integer $C$; and a set of pairs of positive integers

$$
\left\{\left(a_{1}, b_{1}\right), \ldots,\left(a_{N}, b_{N}\right)\right\}
$$

where $a_{i} \leq b_{i}$ for all $1 \leq i \leq N$.

Question: does there exist a partition $S=S_{1} \cup \ldots \cup S_{N}$ such that, for each $1 \leq i \leq N$, either $S_{i}=\emptyset$ or $a_{i} \leq\left|S_{i}\right| \leq b_{i}$, and

$$
\sum_{1 \leq i \leq N} \sum_{\substack{\{j, g\} \subseteq S_{i} \\ j \neq g}} c(j, g) \leq C ?
$$

It is easy to see that the C_PARTITION problem is a decision version of a particular case of the partitioning problem considered in this paper. Therefore, 
the NP-completeness in the strong sense of C_PARTITION implies the NPhardness in the strong sense of the original partitioning problem. The proof is a polynomial reduction to C_PARTITION the following problem:

CLIQUE

Input: undirected graph $G(V, E)$ and positive integer $k$

150 Question: does $G(V, E)$ contain a complete subgraph of $k$ nodes?

It is well known that the CLIQUE problem is NP-complete in the strong sense. [17].

Theorem 1. CLIQUE $\propto C_{-} P A R T I T I O N$ and therefore $C_{-} P A R T I T I O N$ is NPcomplete in the strong sense.

Proof. For any instance of CLIQUE with graph $G(V, E)$ and integer $k$, the corresponding instance of C_PARTITION is constructed as follows:

- $S=V$,

- $N=|V|-k+1$,

- $C=0$,

160

- $a_{i}=b_{i}=1$ for $1 \leq i<N$ and $a_{N}=b_{N}=k$,

- $c(j, g)=\left\{\begin{array}{ll}0, & \text { if }[j, g] \in E \\ 1, & \text { otherwise }\end{array}\right.$.

Then, for any feasible partition, $\left|S_{N}\right|=k$ and $\left|S_{i}\right|=1$ for all $1 \leq i<N$. Hence,

$$
\sum_{1 \leq i \leq N} \sum_{\substack{\{j, g\} \subseteq S_{i} \\ j \neq g}} c(j, g)=\sum_{\substack{\{j, g\} \subseteq S_{N} \\ j \neq g}} c(j, g) \leq 0
$$

if and only if $G(V, E)$ contains a complete subgraph of $k$ nodes. 


\section{Lagrangian Relaxation}

We can formulate the Lagrangian relaxation model by moving the constraints (2) to the objective function:

(QR) Minimise: $\alpha \sum_{i=1}^{N} \sum_{k=1}^{K} \sum_{l=1}^{K} b_{k, l} Y_{i, k} Y_{i, l}+\beta \sum_{i=1}^{N} \sum_{j=1}^{M} c_{i, j} X_{i, j}+\sum_{j=1}^{M} \lambda_{j}\left(1-\sum_{i=1}^{N} X_{i, j}\right)$

Subject To: $\quad(3)-(9)$

where $\lambda_{j} \in \mathbb{R}, j=1, \ldots, M$, is the Lagrangian multiplier corresponding to the

$j$ th constraint (2), which can be interpreted as the penalty of not assigning the $j$ th student to exactly one class. The quadratic term in 15 can be linearised in the same way as with (1).

In (QP), only constraints 2 couple together the $N$ sub-problems of assigning students to a particular class $i$. In (QR), these constraints are moved to 170 the objective function, therefore it is possible to express (QR) as $N$ smaller, class-specific sub-problems $\left(\mathrm{QR}_{i}\right)$ :

$$
\begin{aligned}
\left(\mathrm{QR}_{i}\right) \text { Minimise: } \alpha \sum_{k=1}^{K} \sum_{l=1}^{K} b_{k, l} Y_{i, k} Y_{i, l}+\beta \sum_{j=1}^{M} c_{i, j} X_{i, j}-\sum_{j=1}^{M} \lambda_{j} X_{i, j} \\
\text { Subject To: } a_{i} Z_{i} \leq \sum_{j=1}^{M} X_{i, j} \leq b_{i} Z_{i} \\
p_{i} Z_{i} \leq \sum_{k=1}^{K} Y_{i, k} \leq q_{i} Z_{i} \\
X_{i, j} \leq Y_{i, k} \quad k=1, \ldots, K ; j \in T_{k} \\
X_{i, j} \in\{0,1\} \quad j=1, \ldots, M \\
Y_{i, k} \in\{0,1\} \quad k=1, \ldots, K \\
Z_{i} \in\{0,1\}
\end{aligned}
$$


where the $N$ solutions to $\left(\mathrm{QR}_{i}\right)$ for $1 \leq i \leq N$ are combined to form the solution to $(\mathrm{QR})$.

The Lagrangian relaxation $(\mathrm{QR})$, as its name suggests, is a relaxation on the original model (QP). That is to say, an optimal solution to (QR) given some vector $\lambda$ gives a lower bound on the optimal objective value to (QP). The Lagrangian dual corresponds to the problem of finding the $\lambda$ vector that gives the tightest lower bound. This vector is denoted as $\lambda^{*}$. It is a well-known result that the optimal solution to the Lagrangian dual gives a lower bound to (QP) that is at least as tight as the lower bound provided by the linear relaxation, i.e. the removal of the integrality constraints, of (QP) [18, [19]. A typical way to solve the Lagrangian dual is by means of the iterative subgradient algorithm, which iteratively updates $\lambda$ using

$$
\lambda^{k+1}=\lambda^{k}+\frac{s^{k} \cdot \epsilon_{k}\left(\eta^{*}-\eta^{k}\right)}{\left\|s^{k}\right\|^{2}}
$$

The value of the Lagrangian multipliers $\lambda$ at the $k$ th iteration is denoted by $\lambda^{k}$. The optimal objective value of the Lagrangian dual problem is given by $\eta^{*}$, and $\eta^{k}$ is the objective value of (QR) at the $k$ th iteration. Since the value of $\eta^{*}$ is generally not known in advance, it is typically estimated by means of some heuristic, which will likely give an underestimation on the true value. Therefore, the positive scaling factor $\epsilon_{k}$ is introduced, which is typically given an initial value of 2 and halved whenever the best-known (tightest) lower bound hasn't been improved in a certain number of iterations [18]. A subgradient $s^{k}$ of the Lagrangian dual at iteration $k$ can be given by

$$
s_{j}^{k}=1-\sum_{i=1}^{N} X_{i, j}^{k} \quad j=1, \ldots, M
$$

where $X_{i, j}^{k}$ are the values of the variables obtained at the $k$ th iteration.

\section{5. Lagrangian Heuristic}

The Lagrangian dual problem from Section 4 is solved by means of the subgradient algorithm. In doing so, one solution to the Lagrangian relaxed 
problem is generated for each iteration. These solutions may be infeasible with respect to the original problem since constraints (2) are relaxed. However, by observing trends in certain features of these solutions, it is possible to impose assumptions on the solutions to the underlying problem that reduce the decision space significantly.

The nature of the assumptions we propose to impose is one in which pairs of students must be assigned to the same class. Naturally, if an assumption is imposed that students $j_{1}$ and $j_{2}$ must be paired together, and that students $j_{2}$ and $j_{3}$ must be paired together, then it follows that students $j_{1}$ and $j_{3}$ must be paired together also. One can represent these assumption in the form of a graph, where each vertex corresponds to a student, and each edge between vertices $j_{u}$ and $j_{v}$ corresponds to a pairing assumption between students $j_{u}$ and $j_{v}$. From the terminology of graph theory, a set of such students that must be assigned together is represented by a clique. Due to the transitive nature of student pairing assumptions in this problem, all such cliques are necessarily disjoint.

One of the parameters of the proposed LR-based heuristic determines whether or not to allow cliques with more than 2 vertices. If these cliques are not permitted, then once student pair $\left\{j_{u}, j_{v}\right\}$ is added to the set of assumptions, no further assumptions may be imposed involving students $j_{u}$ or $j_{v}$.

Another parameter of the proposed heuristic is $\rho \in\{0, \ldots, M\}$, which defines the number of explicit pairs to impose. For example, if the pairs $\left\{j_{1}, j_{2}\right\}$ and $\left\{j_{2}, j_{3}\right\}$ are imposed, then it is not necessary to explicitly impose the pair $\left\{j_{1}, j_{3}\right\}$ as it will be implicitly satisfied.

During the subgradient algorithm to compute the Lagrangian dual, many partial solutions are generated, and these are added to a solution pool. For all pairs of students $\left\{j_{u}, j_{v}\right\}$, where $u<v$, each solution in the pool is examined. The number of solutions where $j_{u}$ and $j_{v}$ are together in the same class is denoted $t_{u, v}$. We impose an assumption that those $\rho$ student pairs $\left\{j_{u}, j_{v}\right\}$ who have the highest $t_{u, v}$ values must be paired together in the final solution. Other ranking criteria can be used to decide which pairs of students must be assigned 
together.

It is important that the imposed assumptions reduce the problem size in such a way that the resulting problem is not more difficult to solve. For example, imposing too many assumptions about paired students could make feasible solutions very difficult to find, impairing the performance of the algorithm. A number of other assumption types are considered in [16, including pairs of student who must be assigned separately. The approach of imposing assumptions where students should be assigned separately is less effective as the problem size grows much larger, such as the test cases considered in this paper, as each such assumption on student pairs $\left\{j_{u}, j_{v}\right\}$ assigned separately requires new constraints $X_{i, j_{u}}+X_{i, j_{v}} \leq 1$ for each class $i$. As the number of classes and students grows very large, the large number of these introduced constraints can have the unwanted affect of hindering the performance of the algorithm.

It is possible to impose a wide variety of assumptions in a similar manner. For example, one can impose restrictions on which classes must run or which classes must not, by examining the frequency with which they run or do not run in the solutions from the subgradient algorithm. Likewise, one can impose restrictions on which student types must be assigned to particular classes and which student types must not. In our experience these other types of assumptions can be quite heavy handed, and subsequently make finding feasible solutions to the underlying problem, subject to the assumptions, difficult. It is a matter for further research to investigate whether these assumptions, or others, can be successfully incorporated into this LR-based heuristic framework.

Denote the set of student pairs who must be assigned to the same class as $\mathscr{T}$. The (QP) model, subject to imposed assumptions, is as follows:

$$
\text { (AQP) Minimise: } \quad \alpha \sum_{i=1}^{N} \sum_{k=1}^{K} \sum_{l=1}^{K} b_{k, l} Y_{i, k} Y_{i, l}+\beta \sum_{i=1}^{N} \sum_{j=1}^{M} c_{i, j} X_{i, j}
$$

Subject To: $\quad(2)-(9)$

$$
X_{i, j_{u}}=X_{i, j_{v}} \quad i=1, \ldots, N ;\left\{j_{u}, j_{v}\right\} \in \mathscr{T}
$$


where (27) imposes the assumptions that student pairs in $\mathscr{T}$ must be assigned

Step 5. If $k$ exceeds the maximum number of iterations, then terminate LD-Subroutine and return $\bar{\eta}$ and $\mathscr{P}$; otherwise set $k:=k+1$ and return to Step 2.

The proposed LR-based Heuristic uses the following parameters: $\rho$ which defines the number of non-implicit pairs to add to $\mathscr{T}$, and a parameter that 260

The (AQP) model above is described with equality constraints 27) for convenience, however the model can easily be modified to contain fewer variables. This can be accomplished by redefining the primary variables $X$ so that they no longer correspond to the assignment of a particular student to a class, but a set of one or more students to a class. Minor alterations must also be made to the objective function and the constraints, including a weight coefficient (corresponding to the number of students represented by the variable) added to constraints (3).

\section{LD-Subroutine:}

Step 1. Initialise the Lagrangian multiplier vector to its starting value $\lambda^{1}:=0$, $\epsilon_{1}$, determine an estimate for $\eta^{*}$ by means of a heuristic, set the tightest lower bound $\bar{\eta}:=-\infty$, initialise the empty solution pool $\mathscr{P}$, and initialise the iteration counter $k:=1$.

Step 2. Construct the $\left(\mathrm{QR}_{i}\right)$ models for $1 \leq i \leq N$, solve them, and combine the $N$ partial solutions to form the complete solution and add it to $\mathscr{P}$. Compute $\lambda^{k+1}$ according to 24 .

Step 3. If the objective value of the complete solution $\eta^{k}$ is greater than $\bar{\eta}$, then set $\bar{\eta}:=\eta^{k}$.

Step 4. If $\bar{\eta}$ has not been improved in the last $\tilde{\epsilon}$ iterations, then $\epsilon_{k+1}:=\frac{1}{2} \epsilon_{k}$. Otherwise, $\epsilon_{k+1}:=\epsilon_{k}$. either permits or forbids cliques with more than 2 vertices.

\section{LR-based Heuristic:}


Step 1. Initialise a set of assumed set of pairs $\mathscr{T}$ as an empty set.

Step 2. Run LD-Subroutine and store the result $\mathscr{P}$. For each pair of students $\left\{j_{u}, j_{v}\right\}$, where $u<v$, count the number of instances $t_{u, v}$ in $\mathscr{P}$ where $j_{u}$ and $j_{v}$ are assigned to the same class.

Step 3. Find the student pair $\left\{j_{u}, j_{v}\right\}$ not already in $\mathscr{T}$, or if only 2 -vertex cliques are permitted then the pair $\left\{j_{u}, j_{v}\right\}$ where neither $j_{u}$ nor $j_{v}$ are present in $\mathscr{T}$, whose corresponding $t_{u, v}$ has the greatest value, and add that pair to $\mathscr{T}$. If $|\mathscr{T}|<\rho$ then repeat Step 3 .

Step 4. Solve (AQP) subject to $\mathscr{T}$, and return the solution.

\section{Genetic Algorithm Based Matheuristic}

Although Genetic Algorithms (GA) are often used in solving the QKP and QMKP [20], 21], 13, these publications on GA are not directly applicable to our problem due to the existence of lower bounds on class sizes. Moreover, the the following IP describes a feasible allocation of anonymous students of each type to each class: 


$$
\begin{aligned}
& \text { (FP) Minimise: } \sum_{i=1}^{N} \sum_{k=1}^{K} \gamma_{i, k} \bar{X}_{i, k} \\
& \text { S.T.: } \quad \sum_{i=1}^{N} \bar{X}_{i, k}=\left|T_{k}\right| \quad k=1, \ldots, K \\
& a_{i} Z_{i} \leq \sum_{k=1}^{K} \bar{X}_{i, k} \leq b_{i} Z_{i} \quad i=1, \ldots, N \\
& p_{i} Z_{i} \leq \sum_{k=1}^{K} Y_{i, k} \leq q_{i} Z_{i} \quad i=1, \ldots, N \\
& \bar{X}_{i, k} \leq\left|T_{k}\right| \times Y_{i, k} \quad i=1, \ldots, N ; k=1, \ldots, K \\
& Y_{i, k} \leq \bar{X}_{i, k} \quad i=1, \ldots, N ; k=1, \ldots, K \\
& \bar{X}_{i, k} \in \mathbb{Z}^{+} \quad i=1, \ldots, N ; k=1, \ldots, K \\
& Y_{i, k} \in\{0,1\} \quad i=1, \ldots, N ; k=1, \ldots, K \\
& Z_{i} \in\{0,1\} \quad i=1, \ldots, N
\end{aligned}
$$

The constraints 29 express the requirement that the total number of stu-

dents assigned for each type is equal to the total number of students of that type. The constraints (30) and (31), analogous to (3) and (4), express the requirements that the number of students and student types, respectively, must fall within the allowed ranges for each running class. The constraints 32 and (33), analogous to (5) and (6), express the requirement that students can only

be assigned to classes if their type is also assigned, and that types can only be assigned to classes if at least one student of that type is also assigned. The constants $\gamma_{i, k}$ are chosen randomly each time (FP) is solved, enabling a diverse range of solutions to be produced.

While the feasibility problem was shown to be NP-complete in Section 3 the instances of (FP) that arise from our real-world-inspired test cases can be solved quickly. For all test cases, including all cases where CPLEX could not solve the original QP problem, CPLEX solved (FP) model in under a second.

Next, students of each type are optimally assigned to classes according to 
the numbers obtained from the solution to (FP). This is possible in polynomial for the initial population, the crossover operator involves two stages. First, (FP) is solved to determine which classes should be run in the child, and the number of students of each type in each class, with the additional restriction that those classes that are run in both parents must run in the child, and those classes 330

Next, students are assigned to classes in numbers specified by the numbers obtained from the solution to (FP). Here, the additional restriction is imposed that those students that are assigned to the same class in both parents must be assigned to that class in the child, and any student whose classes in one or both 
in the child. The remaining students are assigned according to the minimum cost network flow approach discussed earlier.

The complete GA algorithm is as follows:

\section{GA-based Matheuristic:}

340 Step 1. Initialise the solution pool $\mathscr{P}$, and the iteration counter $k:=1$.

Step 2. Solve (FP) given random coefficients $\gamma_{i, k}$. Construct a solution to the studied problem by randomly assigning students to classes according to the numbers in the obtained solution to (FP). Add the constructed solution to $\mathscr{P}$. If the $\mathscr{P}$ has not yet reached the required size, repeat Step 2.

Step 3. Pick $n$ solutions at random from $\mathscr{P}$, and identify a solution with the worst objective value. Delete this solution from $\mathscr{P}$.

Step 4. Pick $n$ solutions at random from $\mathscr{P}$, and identify a solution with the best objective value. Denote this solution $\mathscr{A}$.

Step 5. Pick $n$ solutions random from $\mathscr{P}$, and identify a solution with the best

Step 6. Find the optimal solution $F^{*}$ to $(\mathrm{FP})$ given random coefficients $\gamma_{i, k}$, with the additional assumptions that $Z_{i}=0$ for all classes $i$ that are not run in either $\mathscr{A}$ or $\mathscr{B}$, and $Z_{i}=1$ for all classes $i$ that are run in both $\mathscr{A}$ and $\mathscr{B}$. Add to $\mathscr{P}$ a new solution constructed by assigning students to classes one at a time as follows:

i. Any student that is assigned to class $i$ in both $\mathscr{A}$ and $\mathscr{B}$ should be assigned to class $i$ in the constructed solution if there is remaining space in the class.

ii. Any student that is assigned to classes $i_{\mathscr{A}}$ in $\mathscr{A}$ and $i_{\mathscr{B}}$ in $\mathscr{B}$, where $i_{\mathscr{A}}$ and/or $i_{\mathscr{B}}$ are running in $F^{*}$, should be assigned to either class $i_{\mathscr{A}}$ or $i_{\mathscr{B}}$ in the constructed solution, as long as the remaining capacity of the class permits. 
iii. Any remaining students are assigned to the remaining spaces in the constructed solution at random, according to the numbers specified in $F^{*}$.

Step 7. Increment the iteration counter $k$. If $k$ has exceeded the iteration limit, or if the total time limit has been reached, then terminate the algorithm returning a solution from $\mathscr{P}$ with the best objective value. Otherwise, return to Step 3.

370

\section{Computational Results}

Using typical training data from an Australian electricity distributor as a template, we randomly generated a set of 27 of test cases $\$$ Each test case had between 512 and 2048 students across 40 student types, and between 32 and 128 classes.

The minimum and maximum student capacities for the classes in each test case were chosen such that the test cases fall into one of three density groups: low density (L), where approximately $25 \%$ of the classes will be needed and the rest will remain empty; medium density (M), where approximately $50 \%$ of the classes will be needed and the rest will remain empty; and high density $(\mathrm{H})$, where approximately $75 \%$ of the classes will be needed and the rest will remain empty.

We used IBM ILOG CPLEX 12.6.3.0 64-bit on an Intel i7-4790K quadcore 4.00Ghz system with 16GB of RAM, running Windows 10 Professional. Our code was written in C\#, and interacted with CPLEX using the IBM ILOG Concert API. We used default CPLEX settings with the exception of time limits. The GA-based matheuristic is probabilistic, therefore we ran it on each of the test cases 10 times. The pseudorandom number generator we used was the MT19937 Mersenne Twister [22. For the weighted objective function, we used $\alpha=\beta=1$. We determined the average amount of time taken for the LR-based

\footnotetext{
${ }^{1}$ All test cases and solution files are available for download from https://goo.gl/q5Z1cx
} 


\begin{tabular}{|c|c|c|c||c|c|c|c||c|c|c|c|}
\hline Case & Classes & Students & Density & Case & Classes & Students & Density & Case & Classes & Students & Density \\
\hline 01 & 32 & 512 & L & 10 & 64 & 512 & L & 19 & 128 & 512 & L \\
\hline 02 & 32 & 512 & M & 11 & 64 & 512 & M & 20 & 128 & 512 & M \\
\hline 03 & 32 & 512 & H & 12 & 64 & 512 & H & 21 & 128 & 512 & H \\
\hline 04 & 32 & 1024 & L & 13 & 64 & 1024 & L & 22 & 128 & 1024 & L \\
\hline 05 & 32 & 1024 & M & 14 & 64 & 1024 & M & 23 & 128 & 1024 & M \\
\hline 06 & 32 & 1024 & H & 15 & 64 & 1024 & H & 24 & 128 & 1024 & H \\
\hline 07 & 32 & 2048 & L & 16 & 64 & 2048 & L & 25 & 128 & 2048 & L \\
\hline 08 & 32 & 2048 & M & 17 & 64 & 2048 & M & 26 & 128 & 2048 & M \\
\hline 09 & 32 & 2048 & H & 18 & 64 & 2048 & H & 27 & 128 & 2048 & H \\
\hline
\end{tabular}

Table 1: The number of classes (Classes), number of students (Students), and density (Density) for the 27 test cases (Case).

heuristic, and permitted the same amount of time for the GA-based matheuristic and for attempting to solve the QP model directly.

The main properties of the test cases are outlined in Table 1. The columns are as follows: the test case identifier (Case), the number of classes $N$ (Classes), The number of students $M$ (Students), and the density groups (Density).

The number of variables (columns) and constraints (rows) in the (QP) and (LQP) models for each test case is given in Table 2 The size of the models is not dependent on the test case density, therefore test cases with the same number of classes and students, but with differing densities, are grouped together. The columns are as follows: the test case identifiers (Cases), the number variables in the corresponding $(\mathrm{QP})$ model $\left(\mathrm{Cols}_{\mathrm{QP}}\right)$, the number constraints in the corresponding (QP) model (Rows ${ }_{\mathrm{QP}}$ ), the number variables in the corresponding (LQP) model (Cols $\mathrm{QP}_{\mathrm{QP}}$ ), and the number constraints in the corresponding (LQP) model $\left(\right.$ Rows $\left._{\mathrm{QP}}\right)$. For these test cases, the linearised models (LQP) have, on average, roughly 1.84 times as many variables, and roughly 3.46 times as many constraints, as their $(\mathrm{QP})$ counterparts.

For the Lagrangian relaxation procedure, the starting $\epsilon$ value is commonly taken to be $\epsilon_{1}=2$ [18]. For our problem, however, we found the procedure did not perform well with this value, especially for the larger test cases where the best bound remained at zero even after hours and several hundred iterations.

During our initial computational experiments, we found that $\epsilon_{1}=0.05$ with $\tilde{\epsilon}=$ 


\begin{tabular}{|c|c|c|c|c|}
\hline Cases & Cols $_{\mathrm{QP}}$ & Rows $_{\mathrm{QP}}$ & Cols $_{\mathrm{IP}}$ & Rows $_{\mathrm{IP}}$ \\
\hline $01,02,03$ & 17697 & 18304 & 42656 & 93184 \\
\hline $04,05,06$ & 34081 & 35200 & 59040 & 110080 \\
\hline $07,08,09$ & 66849 & 68992 & 91808 & 143872 \\
\hline $10,11,12$ & 35393 & 36096 & 85312 & 185856 \\
\hline $13,14,15$ & 68161 & 69376 & 118080 & 219136 \\
\hline $16,17,18$ & 133697 & 135936 & 183616 & 285696 \\
\hline $19,20,21$ & 70785 & 71680 & 170624 & 371200 \\
\hline $22,23,24$ & 136321 & 137728 & 236160 & 437248 \\
\hline $25,26,27$ & 267393 & 269824 & 367232 & 569344 \\
\hline
\end{tabular}

Table 2: The number of columns (Cols) and rows (Rows) for the QP model (QP) and LQP model (IP) for the 27 test cases (Cases).

2 produced much better performance for the Lagrangian relaxation procedure across all our test cases, however for the purposed of our LR-based heuristic, it is better for the Lagrangian relaxation procedure to generate a large number of diverse solutions than to have fast LR convergence with a small set of solutions.

${ }_{415}$ We therefore used a starting value of $\epsilon_{1}=0.1$ and set $\tilde{\epsilon}$ to 10 .

Table 3 shows a comparison of lower bounds provided by linear relaxation of (LQP) to the Lagrangian relaxation. The columns are as follows: the test case identifier (Case), the linear relaxation bound (LinRel), and the Lagrangian relaxation bound (LagRel). The Lagrangian relaxation provided a lower bound to the objective value of the underlying problem that was between $1 \%$ and $132 \%$, with an average of $36 \%$, higher than the linear relaxation bound.

Table 4 shows a comparison of time required, in seconds, to compute the lower bounds shown in Table 3 . The columns are identical to those in Table 3 The Lagrangian relaxation procedure took between 2.5 and 816, with an average 425 of 103, times longer than the linear relaxation. The Lagrangian relaxation procedure has the added benefit that each iteration produces one feasible partial solution for each class. 


\begin{tabular}{|c|c|c||c|c|c||c|c|c|}
\hline Case & LinRel & LagRel & Case & LinRel & LagRel & Case & LinRel & LagRel \\
\hline 01 & 25035.50 & 26143.16 & 10 & 7258.90 & 9649.33 & 19 & 2812.70 & 5666.28 \\
\hline 02 & 12097.10 & 13628.89 & 11 & 5102.90 & 7566.36 & 20 & 1564.40 & 3632.29 \\
\hline 03 & 12666.40 & 14002.61 & 12 & 3662.90 & 5325.06 & 21 & 1666.50 & 2949.20 \\
\hline 04 & 62811.90 & 63444.30 & 13 & 21060.30 & 23194.41 & 22 & 7232.50 & 10842.10 \\
\hline 05 & 30338.50 & 31758.52 & 14 & 10892.80 & 13587.90 & 23 & 3917.00 & 7765.47 \\
\hline 06 & 22712.00 & 24404.62 & 15 & 11109.40 & 14069.49 & 24 & 2952.60 & 6476.81 \\
\hline 07 & 93526.70 & 94673.71 & 16 & 43627.00 & 45856.09 & 25 & 20935.30 & 24713.55 \\
\hline 08 & 62690.60 & 64573.21 & 17 & 24609.50 & 27704.03 & 26 & 11147.10 & 15749.39 \\
\hline 09 & 49709.40 & 51102.07 & 18 & 18517.30 & 22071.88 & 27 & 6998.30 & 11906.54 \\
\hline
\end{tabular}

Table 3: The lower bound provided by the linear relaxation of LQP (LinRel), and the Lagrangian relaxation bound (LagRel) for the 27 test cases (Case).

\begin{tabular}{|c|c|c||c|c|c||c|c|c|}
\hline Case & LinRel & LagRel & Case & LinRel & LagRel & Case & LinRel & LagRel \\
\hline 01 & 13 & 627 & 10 & 152 & 2023 & 19 & 29 & 2420 \\
\hline 02 & 19 & 1036 & 11 & 16 & 1809 & 20 & 3 & 2447 \\
\hline 03 & 24 & 956 & 12 & 10 & 1612 & 21 & 4 & 2421 \\
\hline 04 & 29 & 857 & 13 & 317 & 1534 & 22 & 630 & 3481 \\
\hline 05 & 34 & 1302 & 14 & 259 & 2539 & 23 & 91 & 4084 \\
\hline 06 & 27 & 1729 & 15 & 80 & 2691 & 24 & 10 & 4610 \\
\hline 07 & 64 & 1544 & 16 & 680 & 2197 & 25 & 1744 & 4370 \\
\hline 08 & 47 & 1726 & 17 & 511 & 3369 & 26 & 572 & 5912 \\
\hline 09 & 75 & 1973 & 18 & 369 & 4040 & 27 & 257 & 5923 \\
\hline
\end{tabular}

Table 4: The time, in seconds, required to compute the linear relation of LQP (LinRel), and the Lagrangian relaxation bound (LagRel) for the 27 test cases (Case). 


\begin{tabular}{|c|c|c|c|c|c|c|}
\hline Case & $10 \% \mathrm{C}$ & $10 \%$ & $20 \% \mathrm{C}$ & $20 \%$ & $30 \% \mathrm{C}$ & $30 \%$ \\
\hline 01 & 27728.0 & 28349.5 & 28260.0 & $\mathbf{2 7 2 2 8 . 5}$ & 30125.0 & 28181.5 \\
\hline 02 & 15512.5 & $\mathbf{1 5 5 1 0 . 5}$ & 15667.0 & 16607.0 & 15952.5 & 15939.0 \\
\hline 03 & $\mathbf{1 5 9 0 1 . 5}$ & 16493.5 & 16027.5 & 16368.5 & 17407.0 & 16821.5 \\
\hline 04 & 156498.0 & 135303.5 & 149792.5 & 68155.5 & $\mathbf{6 5 6 2 4 . 5}$ & 152989.0 \\
\hline 05 & 113635.5 & 102934.5 & 161448.0 & 34396.5 & 37772.5 & $\mathbf{3 3 4 6 8 . 5}$ \\
\hline 06 & 26498.5 & 28689.5 & $\mathbf{2 5 9 1 4 . 0}$ & 26448.0 & 29558.0 & 26252.0 \\
\hline 07 & 284443.5 & 264407.0 & 337967.0 & $\mathbf{9 6 7 7 7 . 0}$ & 310202.5 & 97785.5 \\
\hline 08 & 71145.5 & 207659.5 & 178323.5 & 235653.5 & $\mathbf{7 0 4 6 4 . 5}$ & 205008.0 \\
\hline 09 & 52939.5 & 161932.5 & 154799.5 & 149034.5 & $\mathbf{5 2 7 7 3 . 5}$ & 53392.5 \\
\hline 10 & 51398.0 & 56282.0 & 83387.5 & 69566.5 & $\mathbf{4 6 4 2 5 . 0}$ & 64256.0 \\
\hline 11 & 54987.0 & 49014.0 & $\mathbf{1 3 4 2 5 . 0}$ & 17713.0 & 14943.5 & 15080.0 \\
\hline 12 & 13189.0 & 11561.0 & 47952.5 & 10607.5 & $\mathbf{8 6 5 0 . 5}$ & 9955.5 \\
\hline 13 & 149670.5 & 111301.5 & 121775.5 & 112428.5 & 160489.5 & $\mathbf{1 0 8 4 3 3 . 0}$ \\
\hline 14 & 16297.0 & 16352.5 & $\mathbf{1 5 9 5 3 . 0}$ & 63331.0 & 16090.0 & 16286.0 \\
\hline 15 & 21600.5 & 29704.0 & 24326.0 & $\mathbf{2 0 9 0 3 . 0}$ & 24969.0 & 21562.5 \\
\hline 16 & 193459.0 & $\mathbf{1 8 4 8 7 9 . 5}$ & 224889.0 & 218679.5 & 200310.5 & 278620.0 \\
\hline 17 & 230104.0 & $\mathbf{1 7 6 4 2 9 . 0}$ & 241710.0 & 250492.0 & 204366.0 & 272560.0 \\
\hline 18 & $\mathbf{2 9 9 4 6 . 0}$ & 320669.5 & 30025.5 & 112569.0 & 269609.5 & 34162.5 \\
\hline 19 & 60118.5 & 65537.0 & 66608.0 & 57081.5 & 59630.0 & $\mathbf{1 8 5 4 0 . 5}$ \\
\hline 20 & 52531.0 & 59937.0 & 77608.5 & $\mathbf{3 5 5 6 4 . 5}$ & 40345.0 & 38385.5 \\
\hline 21 & $\mathbf{8 5 5 0 1 . 5}$ & 86071.0 & 86321.0 & 96603.0 & 96240.0 & 90446.5 \\
\hline 22 & 107470.5 & 76535.0 & $\mathbf{6 7 5 4 5 . 0}$ & 117384.0 & 83727.5 & 100723.5 \\
\hline 23 & $\mathbf{1 0 2 9 2 4 . 5}$ & 123642.0 & 116893.5 & 149978.0 & 130494.0 & 115310.0 \\
\hline 24 & 84763.5 & 90099.0 & 160378.5 & $\mathbf{7 4 0 0 2 . 0}$ & 92816.5 & 78813.0 \\
\hline 25 & 245627.0 & $\mathbf{1 8 6 0 0 2 . 5}$ & 190062.0 & 218624.0 & 240426.0 & 243105.0 \\
\hline 26 & 291573.5 & 331949.0 & 310532.5 & 318246.0 & 285952.5 & $\mathbf{2 4 3 2 0 0 . 5}$ \\
\hline 27 & $\mathbf{2 4 7 3 3 2 . 5}$ & 320109.5 & 260695.0 & 274399.0 & 276121.5 & 312599.0 \\
\hline
\end{tabular}

Table 5: Objective values from the LR-based heuristic using $10 \%$ of students paired with and without large cliques $(10 \% \mathrm{C})$ and $(10 \%), 20 \%$ of students paired with and without large cliques $(20 \% \mathrm{C})$ and $(20 \%)$, and $30 \%$ of students paired with and without large cliques $(30 \%$ C) and (30\%) for the 27 test cases (Case). 
For the LR-based heuristic, we investigate $\rho$ values corresponding to $10 \%$, $20 \%$, and $30 \%$ of the total population size, and we investigate the effect where cliques with more than two vertices are either permitted or are forbidden. In all cases, the time limit, in seconds, to solve the AQP model was equal to the number of students. This time limit was reached for all test cases. Table 5 shows the objective values of the solutions produced using the LR-based heuristic. The columns are as follows: The test case identifier (Case), $\rho$ set to $10 \%$ of the population size with larger cliques permitted $(10 \% \mathrm{C})$, and with larger cliques forbidden (10\%), $\rho$ set to $20 \%$ of the population size with larger cliques permitted $(20 \% \mathrm{C})$, and with larger cliques forbidden $(20 \%), \rho$ set to $30 \%$ of the population size with larger cliques permitted $(30 \% \mathrm{C})$, and with larger cliques forbidden (30\%).

The $(10 \% \mathrm{C})$ produced the best solutions for 5 test cases, (10\%) for 4 test cases, $(20 \% \mathrm{C})$ for 4 test cases, $(20 \%)$ for 5 test cases, $(30 \% \mathrm{C})$ for 5 test cases, and (30\%) for 4 test cases. The runs where larger cliques were permitted produced best solutions for 14 test cases, and produced best solutions for 13 test cases where larger cliques were forbidden. When looking at the ratio between the objective value of the solution produced given certain parameters to the objective value of the best solution produced, (10\%) performed the worst, with a ratio of about 1.996 , while $(30 \%)$ performed the best, with a ratio of about 1.248. Overall, the ratio where large cliques were permitted was about 1.649, and the ratio was about 1.609 when large cliques were forbidden. According to these results, it appears that the selecting the largest of the tested values of $\rho$ and forbidding large cliques had the best outcome overall. This is most likely because imposing more student pairs allowed the solver to find solutions to the problem with imposed pairs faster, and because forbidding large cliques would result in a greater variety of students present in imposed pairs for a given value 455 of $\rho$.

For the GA-based matheuristic, we explored the tournament size for the $n$-tournament selection operator, $n=\{N, 5 N, 10 N\}$, with a fixed population size of $100 N$. In all cases, the GA-based matheuristic was permitted to run for 
an arbitrary number of iterations, but had a time limit equal to the average amount of time taken for the LR-based heuristic for that test case.

We also tested variations on the $n$-tournament selection operators, in which parent solutions are selected as inputs for the crossover operator. The three variations for selecting the parents were

1. Best: the solution with the best objective value from a tournament of size $n$;

2. Random: a random solution ${ }^{2}$ from a tournament of size $n$; and

3. Worst: the solution with the worst objective value from a tournament of size $n$.

The selection operator is invoked twice in order to select two parents. We compared the relative performance of selection pairs $\{$ Best, Best\}, \{Best, Random\}, and \{Best, Worst\}.

In all cases tested, \{Best, Best\} produced consistently superior results. \{Best, Random\} produced final solutions that were, on average, $11.752 \%$ worse than $\{$ Best, Best\}, and \{Best, Worst \} produced final solutions that were, on 475 average, $22.727 \%$ worse than $\{$ Best, Best\}. Since $\{$ Best, Best $\}$ produced consistently superior solutions, we do not report the results of \{Best, Random $\}$ and \{Best, Worst $\}$ in the remainder of the paper.

Table 6 shows a comparison of the results of the GA-based matheuristic using different tournament sizes. The columns are as follows: the test case identifier (Case), followed by the minimum (Min), average (Avg), and maximum (Max) objective value reported across the 10 runs of the GA-based matheuristic using the different population sizes. For each test case, the parameter set that produced the best results on average are indicated. The results clearly show that using larger tournament sizes results for the GA-based matheuristic produces 485 the best results overall. Using $n=5 N$ results in final solutions with objective

\footnotetext{
${ }^{2}$ Note that Random is equivalent to selecting a random solution from the entire population.
} 


\begin{tabular}{|c|c|c|c|c|c|c|c|c|c|}
\hline Case & $N$ Min & $N$ Avg & $N \operatorname{Max}$ & $5 N$ Min & $5 N \mathrm{Avg}$ & $5 N \operatorname{Max}$ & $10 N$ Min & $10 N \mathrm{Avg}$ & $10 N \operatorname{Max}$ \\
\hline 01 & 91242.0 & 104088.8 & 126507.0 & 89317.0 & 100309.4 & 107197.5 & 70969.0 & 96212.6 & 106917.5 \\
\hline 02 & 80001.5 & 87148.5 & 101027.0 & 78458.0 & 84956.9 & 97735.5 & 71913.5 & 81374.2 & 87640.0 \\
\hline 03 & 67970.5 & 73771.3 & 83557.5 & 64769.5 & 67121.1 & 68708.0 & 57838.5 & 67702.6 & 73492.5 \\
\hline 04 & 186384.5 & 212467.4 & 243732.0 & 163696.5 & 186075.5 & 199798.0 & 134469.0 & 172069.1 & 207858.0 \\
\hline 05 & 152188.5 & 181547.4 & 209962.5 & 166769.0 & 173756.5 & 181388.0 & 127361.5 & 158909.7 & 187397.0 \\
\hline 06 & 139030.0 & 152711.7 & 176796.5 & 126221.0 & 144869.3 & 156351.0 & 114863.5 & 132484.3 & 150388.5 \\
\hline 07 & 339135.5 & 431919.6 & 483614.0 & 373937.0 & 412425.4 & 450458.0 & 292499.0 & 362869.7 & 455445.5 \\
\hline 08 & 345296.5 & 379839.0 & 416811.0 & 332903.5 & 355297.7 & 374316.0 & 270846.5 & 336157.9 & 373108.0 \\
\hline 09 & 229166.5 & 247770.2 & 258267.5 & 207775.5 & 224390.2 & 260741.5 & 200377.5 & 238324.4 & 272314.0 \\
\hline 10 & 65488.0 & 76016.5 & 87723.0 & 54077.5 & 72858.1 & 81155.5 & 54570.5 & 70972.1 & 84968.5 \\
\hline 11 & 62119.5 & 67928.3 & 77767.0 & 58894.0 & 63194.5 & 68366.5 & 51782.5 & 59756.5 & 67927.5 \\
\hline 12 & 67654.0 & 77697.6 & 85777.0 & 68634.0 & 72986.8 & 76799.0 & 63146.0 & 69697.7 & 77014.5 \\
\hline 13 & 127503.5 & 159373.0 & 191844.0 & 134490.5 & 145765.4 & 159078.0 & 127183.0 & 150736.9 & 184762.5 \\
\hline 14 & 107942.0 & 121064.8 & 133317.5 & 99062.0 & 110802.5 & 126008.0 & 86389.0 & 104101.8 & 117581.0 \\
\hline 15 & 113694.5 & 133210.4 & 150128.5 & 120876.5 & 133918.6 & 143327.5 & 95478.5 & 119608.7 & 153496.5 \\
\hline 16 & 276536.5 & 328791.5 & 372141.0 & 275411.0 & 306524.9 & 339932.5 & 241305.0 & 283971.6 & 312162.5 \\
\hline 17 & 218382.0 & 268251.9 & 296087.5 & 224007.0 & 246367.0 & 275521.0 & 204612.0 & 233492.8 & 262544.0 \\
\hline 18 & 238596.5 & 262473.8 & 309772.5 & 231984.0 & 263037.9 & 284629.0 & 207467.0 & 246838.2 & 287351.5 \\
\hline 19 & 55876.5 & 69762.7 & 82040.0 & 44653.5 & 64460.8 & 85016.5 & 48913.5 & 57696.7 & 67454.0 \\
\hline 20 & 48163.5 & 53883.0 & 66689.0 & 48211.0 & 52363.9 & 57575.0 & 39044.0 & 45617.2 & 55981.5 \\
\hline 21 & 41338.0 & 75161.5 & 92132.5 & 37948.0 & 57068.9 & 82655.5 & 40752.0 & 53276.6 & 88666.5 \\
\hline 22 & 129922.5 & 147397.9 & 163537.0 & 135758.0 & 152261.8 & 175112.5 & 101933.0 & 130587.5 & 150005.0 \\
\hline 23 & 119509.0 & 135456.7 & 152733.5 & 106466.5 & 130127.5 & 145559.5 & 94491.0 & 120684.6 & 144671.0 \\
\hline 24 & 111839.0 & 137977.1 & 170726.5 & 114220.0 & 124454.0 & 134612.5 & 76647.5 & 110887.4 & 130358.0 \\
\hline 25 & 260619.5 & 305671.7 & 350736.0 & 261987.0 & 291420.2 & 324293.5 & 222027.0 & 275145.5 & 340572.0 \\
\hline 26 & 201494.5 & 252628.5 & 284579.0 & 212065.0 & 233238.4 & 251600.0 & 188994.0 & 228316.5 & 249142.0 \\
\hline 27 & 124748.0 & 223403.6 & 259918.5 & 119727.0 & 211191.7 & 241518.0 & 136182.5 & 181289.6 & 211825.5 \\
\hline
\end{tabular}

Table 6: Minimum (Min), Average (Avg), and Maximum (Max) objective value for GA-based matheuristic with tournament sizes $N, 5 N$, and $10 N$ for the 27 test cases (Case). 
values that are, on average, $6.98 \%$ worse than $n=10 N$, and using $n=N$ results in final solutions with objective values that are, on average, $13.81 \%$ worse than $n=10 N$. Using larger tournament sizes increases the likelihood that better solutions will be chosen from the population, however the selection procedure becomes slightly more time intensive. Using a very large tournament size may not always be advisable, however, as it may decrease the genetic diversity in the population due to the same few good solutions being selected in most iterations for crossover.

Table 7 shows the solution quality of the LR-based heuristic, GA-based matheuristic, and straightforward approach of solving (QP). To solve (QP), CPLEX was allowed the same time limit the GA-based matheuristic was allowed, which was equal to the average amount of time taken by the LR-based heuristic. The columns are as follows: the test case identifier (Case), the objective value from the LR-based heuristic using the parameter set that performed the poorest overall (LR Max), the average objective value using the LR-based heuristic (LR Avg), the objective value from the LR-based heuristic using the parameter set that performed the best overall (LR Min), the objective value from the GA-based matheuristic using the parameter set that performed the poorest overall (GA Max), the average objective value using the GA-based matheuristic 505 (GA Avg), the objective value from the GA-based matheuristic using the parameter set that performed the best overall (GA Min), and the objective value using the straightforward approach of solving (QP).

The GA-based matheuristic showed worse performance than the LR-based heuristic in all but four test cases: 20,21, 26, and 27. The best reported solution values for the GA-based matheuristic were also worse than those from the LRbased heuristic in all but four test cases: 21, 23, 26, and 27. These results might suggest that the GA-base matheuristic is a more promising approach as the problem size grows larger. In order to test this hypothesis, we generated an additional three test cases that are much larger than the previous 27 (See 515 Table 8).

The additional test cases, outlined in Table 8 , require 1.2 million variables 


\begin{tabular}{|c|c|c|c|c|c|c|c|}
\hline Case & LR Max & LR Avg & LR Min & GA Max & GA Avg & GA Min & $\mathrm{QP}$ \\
\hline 01 & 28349.5 & 28312.1 & 28181.5 & 126507.0 & 100203.6 & 70969.0 & 86540.5 \\
\hline 02 & 15510.5 & 15864.8 & 15939.0 & 101027.0 & 84493.2 & 71913.5 & 86955.5 \\
\hline 03 & 16493.5 & 16503.3 & 16821.5 & 83557.5 & 69531.7 & 57838.5 & 58051.0 \\
\hline 04 & 135303.5 & 121393.8 & 152989.0 & 243732.0 & 190204.0 & 134469.0 & - \\
\hline 05 & 102934.5 & 80609.3 & 33468.5 & 209962.5 & 171404.5 & 127361.5 & 140778.5 \\
\hline 06 & 28689.5 & 27226.7 & 26252.0 & 176796.5 & 143355.1 & 114863.5 & 81185.5 \\
\hline 07 & 264407.0 & 231930.4 & 97785.5 & 483614.0 & 402404.9 & 292499.0 & - \\
\hline 08 & 207659.5 & 161375.8 & 205008.0 & 416811.0 & 357098.2 & 270846.5 & - \\
\hline 09 & 161932.5 & 104145.3 & 53392.5 & 258267.5 & 236828.3 & 200377.5 & - \\
\hline 10 & 56282.0 & 61885.8 & 64256.0 & 87723.0 & 73282.2 & 54570.5 & 65036.5 \\
\hline 11 & 49014.0 & 27527.1 & 15080.0 & 77767.0 & 63626.4 & 51782.5 & 68323.0 \\
\hline 12 & 11561.0 & 16986.0 & 9955.5 & 85777.0 & 73460.7 & 63146.0 & 60527.5 \\
\hline 13 & 111301.5 & 127349.8 & 108433.0 & 191844.0 & 151958.4 & 127183.0 & 116016.0 \\
\hline 14 & 16352.5 & 24051.6 & 16286.0 & 133317.5 & 111989.7 & 86389.0 & 138667.5 \\
\hline 15 & 29704.0 & 23844.2 & 21562.5 & 150128.5 & 128912.6 & 95478.5 & 109096.5 \\
\hline 16 & 184879.5 & 216806.3 & 278620.0 & 372141.0 & 306429.3 & 241305.0 & 257972.5 \\
\hline 17 & 176429.0 & 229276.8 & 272560.0 & 296087.5 & 249370.6 & 204612.0 & 161865.5 \\
\hline 18 & 320669.5 & 132830.3 & 34162.5 & 309772.5 & 257450.0 & 207467.0 & 190473.5 \\
\hline 19 & 65537.0 & 54585.9 & 18540.5 & 82040.0 & 63973.4 & 48913.5 & 49032.0 \\
\hline 20 & 59937.0 & 50728.6 & 38385.5 & 66689.0 & 50621.4 & 39044.0 & 54659.5 \\
\hline 21 & 86071.0 & 90197.2 & 90446.5 & 92132.5 & 61835.7 & 40752.0 & 96164.5 \\
\hline 22 & 76535.0 & 92230.9 & 100723.5 & 163537.0 & 143415.7 & 101933.0 & 134996.0 \\
\hline 23 & 123642.0 & 123207.0 & 115310.0 & 152733.5 & 128756.3 & 94491.0 & 135128.5 \\
\hline 24 & 90099.0 & 96812.1 & 78813.0 & 170726.5 & 124439.5 & 76647.5 & 116918.5 \\
\hline 25 & 186002.5 & 220641.1 & 243105.0 & 350736.0 & 290745.8 & 222027.0 & - \\
\hline 26 & 331949.0 & 296909.0 & 243200.5 & 284579.0 & 238061.1 & 188994.0 & 186099.0 \\
\hline 27 & 320109.5 & 281876.1 & 312599.0 & 259918.5 & 205295.0 & 136182.5 & 241980.5 \\
\hline
\end{tabular}

Table 7: Poorest (Max), average (Avg), and best (Min) objective values from the LR-based heuristic (LR), the GA-based matheuristic (GA), and QP model (QP) for the 27 test cases (Case). 


\begin{tabular}{|c|c|c|c|}
\hline Case & Classes & Students & Density \\
\hline 28 & 384 & 3072 & L \\
\hline 29 & 384 & 3072 & M \\
\hline 30 & 384 & 3072 & H \\
\hline
\end{tabular}

Table 8: The number of classes (Classes), number of students (Students), and density (Density) for the three additional test cases (Case).

\begin{tabular}{|c|c|c|}
\hline Case & LR & GA \\
\hline 28 & 90563.5 & 79109.0 \\
\hline 29 & 91979.0 & 70907.0 \\
\hline 30 & 103595.5 & 190749.0 \\
\hline
\end{tabular}

Table 9: The objective values from the LR-based heuristic (LR) and the GA-based matheuristic for the three additional test cases (Case).

and 1.2 million constraints using the (AQP) formulation, or 1.5 million variables and 2.1 million constraints using the (LQP) formulation. For these additional test cases, even finding the linear relaxation proved to be computationally very challenging, taking nearly four hours, therefore we did not attempt to solve these harder cases using direct application of mathematical programming.

We first solved the additional test cases using the LR-based heuristic, and then by the GA-based matheuristic, where the GA-based approach was allowed a time limit equal to the time taken by the LR-based procedure. Table 9 shows the solution values provided by the two methods. Overall, the GA-based approach produced solutions with objective values about $16 \%$ smaller than those produced by the LR-based approach.

A suggested explanation for why the GA-based approach appears to be more promising for larger test cases is that the final step of the LR-based approach is 530 to solve the (AQP) model subject to the imposed pairs. The difficulty of solving this model, even with students fixed in pairs, grows rapidly as the problem size increases. Increasing the number of fixed pairs will likely result in a more tractable model, however the the additional constraints imposed may poorly 
affect the final solution quality.

This paper considered the Lagrangian relaxation-based heuristic and Genetic algorithm-based matheuristic separately, however there are several papers in the literature that report success from hybridising Lagrangian relaxation with

This paper studied a partitioning problem, which we denote "Partition into Inhomogeneous Classes" (PIC). One application of this problem, and the motivation for this research, is the problem of class formation for training and retraining sessions at large electricity distributors.

Two different solution approaches were developed. One is based on the Quadratic Multiple Knapsack formulation and Lagrangian relaxation. The other is a matheuristic developed as an amalgamation of Genetic Algorithms and Integer Programming.

The two solution approaches, as well as the direct solution of the Quadratic Multiple Knapsack formulation, are validated and compared by means of computational experimentation. The test cases were randomly generated using typical training data from an Australian electricity distributor as a template. The Lagrangian relaxation based heuristic performs the best on most of the test cases, whereas the Genetic Algorithm based matheuristic was superior on some of the larger test cases. The superiority of the Genetic Algorithm based approach for larger test cases was further shown experimentally by results on three additional larger test cases. The approach of solving the Quadratic Programming model produced better results than the two proposed solution approaches for 9 of the 27 test cases, however failed to produce any feasible solutions for 5 of the test cases. Based on the results of these computational experiments, both the Lagrangian relaxation based heuristic and Genetic Algorithm based matheuristic performed better than direct application of mathematical programming, and the Genetic Algorithm based approach performed best on the problems of larger size. 
genetic algorithms, such as [23, [24, 25]. Future research should investigate whether such Lagrangian relaxation and genetic algorithm hybrid approaches can produce good results for this problem.

\section{References}

[1] A. Buluç, H. Meyerhenke, I. Safro, P. Sanders, C. Schulz, Recent advances in graph partitioning, CoRR, abs/1311.3144.

[2] E. L. Johnson, A. Mehrotra, G. L. Nemhauser, Min-cut clustering, Mathematical programming 62 (1-3) (1993) 133-151.

[3] K. Andreev, H. Racke, Balanced graph partitioning, Theory of Computing Systems 39 (6) (2006) 929-939.

[4] C.-E. Bichot, Metaheuristics versus spectral and multilevel methods applied on an air traffic control problem, in: International Federation of Automatic Control, Citeseer, 2006.

[5] A. B. Kahng, J. Lienig, I. L. Markov, J. Hu, VLSI physical design: from graph partitioning to timing closure, Springer Science \& Business Media, 2011.

[6] H. Li, G. W. Rosenwald, J. Jung, C.-C. Liu, Strategic power infrastructure defense, Proceedings of the IEEE 93 (5) (2005) 918-933.

[7] J. Li, C.-C. Liu, Power system reconfiguration based on multilevel graph partitioning, in: PowerTech, 2009 IEEE Bucharest, IEEE, 2009, pp. 1-5.

[8] U. Lauther, An extremely fast, exact algorithm for finding shortest paths in static networks with geographical background, Geoinformation und Mobilität-von der Forschung zur praktischen Anwendung 22 (2004) 219-230.

[9] D. Luxen, D. Schieferdecker, Candidate sets for alternative routes in road networks, Journal of Experimental Algorithmics (JEA) 19 (2015) 2-7. 
[10] S. Chopra, M. R. Rao, The partition problem, Mathematical Programming $59(1-3)(1993) 87-115$.

[11] Y. Chen, J.-K. Hao, Iterated responsive threshold search for the quadratic multiple knapsack problem, Ann Oper Res 226 (2015) 101-131.

[12] C. García-Martínez, F. Rodriguez, M. Lozano, Tabu-enhanced iterated greedy algorithm: A case study in the quadratic multiple knapsack problem, European Journal of Operational Research 232 (2014) 454-463.

[13] A. Hiley, B. A. Julstrom, The quadratic multiple knapsack problem and three heuristic approaches to it, in: Proceedings of the 8th annual conference on Genetic and evolutionary computation, ACM, 2006, pp. 547-552.

[14] B. A. Julstrom, Greedy, genetic, and greedy genetic algorithms for the quadratic knapsack problem, in: Proceedings of the 7th annual conference on Genetic and evolutionary computation, ACM, 2005, pp. 607-614.

[15] A. Caprara, D. Pisinger, P. Toth, Exact solution of the quadratic knapsack problem, INFORMS Journal on Computing 11 (2) (1999) 125-137.

[16] O. G. Czibula, H. Gu, Y. Zinder, A lagrangian relaxation-based heuristic to solve large extended graph partitioning problems, in: WALCOM: Algorithms and Computation, Springer, 2016, pp. 327-338.

[17] M. R. Garey, D. S. Johnson, Computers and intractibility (1979).

[18] M. Guignard, Lagrangean relaxation, Top 11 (2) (2003) 151-200.

[19] M. L. Fisher, The lagrangian relaxation method for solving integer programming problems, Management science 50 (12_supplement) (2004) 18611871.

[20] T. Saraç, A. Sipahioglu, A genetic algorithm for the quadratic multiple knapsack problem, in: Advances in Brain, Vision, and Artificial Intelligence, Springer, 2007, pp. 490-498. 
[21] A. Singh, A. S. Baghel, A new grouping genetic algorithm for the quadratic multiple knapsack problem, in: Evolutionary Computation in Combinatorial Optimization, Springer, 2007, pp. 210-218.

[22] M. Matsumoto, T. Nishimura, Mersenne twister: a 623-dimensionally equidistributed uniform pseudo-random number generator, ACM Transactions on Modeling and Computer Simulation (TOMACS) 8 (1) (1998) 3-30.

[23] S. Orero, M. Irving, A combination of the genetic algorithm and lagrangian relaxation decomposition techniques for the generation unit commitment problem, Electric Power Systems Research 43 (3) (1997) 149-156.

[24] X. Zhang, J. Zhao, X. Chen, A hybrid method of lagrangian relaxation and genetic algorithm for solving uc problem, in: 2009 International Conference on Sustainable Power Generation and Supply, IEEE, 2009, pp. 1-6.

[25] L. Nepomuceno, E. C. Baptista, A. R. Balbo, E. M. Soler, Coevolutionary genetic algorithm based on the augmented lagrangian function for solving the economic dispatch problem, IEEE Latin America Transactions 13 (10) (2015) 3277-3286. 